\title{
Adaptive Tutoring in an Intelligent Conversational Agent System
}

\author{
Annabel Latham ${ }^{1}$, Keeley Crockett ${ }^{1}$, David McLean ${ }^{1}$ and Bruce Edmonds ${ }^{2}$ \\ ${ }^{1}$ Intelligent Systems Group, School of Computing, Mathematics \& Digital Technology, \\ Manchester Metropolitan University, Manchester M1 5GD, UK \\ ${ }^{2}$ Centre for Policy Modelling, Manchester Metropolitan University, Aytoun Building, Aytoun \\ Street, Manchester M1 3GH, UK \\ \{A.Latham, K.Crockett, D.McLean, B.Edmonds\} @mmu.ac.uk
}

\begin{abstract}
This paper describes an adaptive online conversational intelligent tutoring system (CITS) called Oscar that delivers a personalised natural language tutorial. During the tutoring conversation, Oscar CITS dynamically predicts and adapts to a student's learning style. Oscar CITS aims to mimic a human tutor by using knowledge of learning styles to adapt its tutoring style and improve the effectiveness of the learning experience. Learners can intuitively explore and discuss topics in natural language, helping to establish a deeper understanding of the topic and boost confidence. An initial study into the adaptation to learning styles is reported which produced encouraging results and positive test score improvements. The results show that students experiencing a tutorial adapted to suit their learning styles performed significantly better than those experiencing an unsuited tutorial.
\end{abstract}

Keywords: Intelligent Tutoring System, Conversational Agent, Adaptive System, Learning Style

\section{Introduction}

The opportunities offered by online learning, both in terms of distance-learning and in supporting traditional classroom activities, have never been more promising. Traditional computerised learning systems which focussed on content delivery have been extended by incorporating intelligent techniques such as adaptation to improve the effectiveness of a learner's experience. Intelligent Tutoring Systems (ITS) personalise tutoring by adapting the learning material delivered according to existing knowledge [1] or student affect such as emotion [2]. ITS that build in some social awareness, such as personalising tutoring to the individual, offer a more familiar and comfortable learning experience. Most ITS are menu-based and offer student-directed study and support at a time and pace to suit individuals, although the experience is more like a computerised book than a classroom tutorial. Conversational Intelligent Tutoring Systems (CITS) incorporate more human-like natural language interfaces 
which allow learners to explore and discuss a topic, supporting the constructivist style of learning used by human tutors. However, few CITS exist that can converse naturally with a learner [3] [4] as their development is complex and labour-intensive. Human tutors use cues they pick up from students to adapt their tutorial content and style, for example indications of the level of existing knowledge and preferred learning styles. Learning styles describe the way groups of people prefer to learn, for example by trial and error or by observation [5]. A CITS that can mimic a human tutor by leading an adaptive tutorial conversation offers students a familiar format that can help improve confidence and motivation, leading to a better learning experience. There are no CITS that can predict and adapt to student learning styles during a tutoring conversation.

This paper describes the adaptation strategy developed for Oscar, a novel CITS that dynamically predicts and adapts to a student's learning style during a tutor-led conversation. Oscar was designed to model a face-to-face tutorial by building in knowledge of learning styles rather than designing an interface specifically to pick up learning style behaviour, as in [6]. The innovative Oscar CITS adaptation algorithm is presented, which uniquely considers both the student's learning style preferences and the opportunity for adaptation in tutoring questions. This approach recognises the importance of providing a coherent learning experience as well as personalisation in tutoring.

In this paper, section 2 introduces the background concepts of Intelligent Tutoring Systems, the Index of Learning Styles, Conversational Agents, Oscar CITS and the Oscar CITS Prediction of Learning Styles. Section 3 describes the adaptive Oscar CITS and its architecture. Section 4 explains the methods used to incorporate adaptivity into the Oscar CITS. Section 5 outlines the experimental methodology and two sample learner dialogues. Section 6 reports the results and discussion, and Section 7 describes the conclusions and future work.

\section{Background}

\subsection{Intelligent Tutoring Systems}

Intelligent tutoring systems (ITS) extend the traditional computerised learning systems 'one-size-fits-all' approach by capturing and modelling individual traits used to personalise the instruction [1]. By applying intelligent systems techniques, ITS aim to improve the effectiveness of tutorials and boost learners' motivation and confidence. Most ITS are menu-based and adapt the tutoring by reordering or ranking menus or hyperlinks to recommend a particular sequence to learners [7]. Such student-directed ITS are little different to recommending particular chapters of a book but conversational interfaces allow a more natural, teacher-led learning experience that supports the constructivist approach favoured by human tutors [8]. AutoTutor [3] and CIRCSIM-tutor [9] are examples of conversational ITS which help students construct knowledge through discussion, however neither adapt to learning styles during tutoring.

There are three main approaches to intelligent tutoring [1]: 
Intelligent solution analysis involves giving students detailed feedback on incomplete or erroneous solutions, helping them learn from their mistakes. In SQLTutor [10] the constraint based modelling technique is used to model the syntax and semantics of SQL. Student solutions are compared to the constraint model and intelligent feedback is given on errors and omissions.

Problem solving support involves intelligent assistance to help learners reach a solution. This approach adopts the constructivist style of teaching, as used by human tutors, to prompt learners to construct their own knowledge and encourage a deeper understanding of a topic. In CIRCSIM-tutor [9], hints are given to help students diagnose physiology problems.

Curriculum sequencing involves adapting the tutoring to deliver learning material in a sequence and style best suited to an individual's needs. Personalised tutoring was originally based on existing knowledge, aiming to improve learning by focussing on topics that are unknown or require improvement [1]. More recently personalisation has been extended to include other individual characteristics that might affect learning, such as the learner's emotions [11], personality [12] or learning style [4], [7]. D'Mello et al. [2] mimicked human tutors in encouraging engagement by adapting to learner's emotions such as boredom or frustration. In order to implement curriculum sequencing, ITS must capture and represent student characteristics in a student model and then use that information as a basis for adaptation.

A minority of ITS incorporate all three techniques as they are complicated and timeconsuming to develop, but the Oscar CITS presented in this paper incorporates all three intelligent technologies by personalising learning material according to learning style and intelligently discussing problems and solutions with students.

\subsection{The Index of Learning Styles}

The Index of Learning Styles (ILS) model [5] was developed to describe the learning styles in engineering education and their associated teaching styles. There are four aspects of learning styles, called dimensions, which correspond to steps in the process of receiving and processing information, as shown in Fig. 1. A student's learning styles are represented as a point on each of the four dimensions to indicate the strength and nature of their learning style preference. Learning style is measured using a 44-question forced-choice self-assessment questionnaire with 11 questions per learning style. For each dimension, answers are compared to result in a learning style and a strength score. The strength score indicates the strength of preference for that learning style, and is used to place the learner on the learning style dimension axis. Low scores place the learner at the centre of the axis and indicate a low preference for that learning style, called neutral preferences. The ILS has 16 (24) combinations of learning styles, for example intuitive/visual/active/global.

For each learning style, the ILS model details typical learner behaviours and preferred teaching styles. This information is beneficial when developing a CITS that can adapt its teaching style to individual learner preferences. 
The ILS model was incorporated into the Oscar CITS as engineering students will make up the initial experimental groups. However the flexible modular structure of the Oscar CITS does not restrict the choice of learning style model to the ILS.

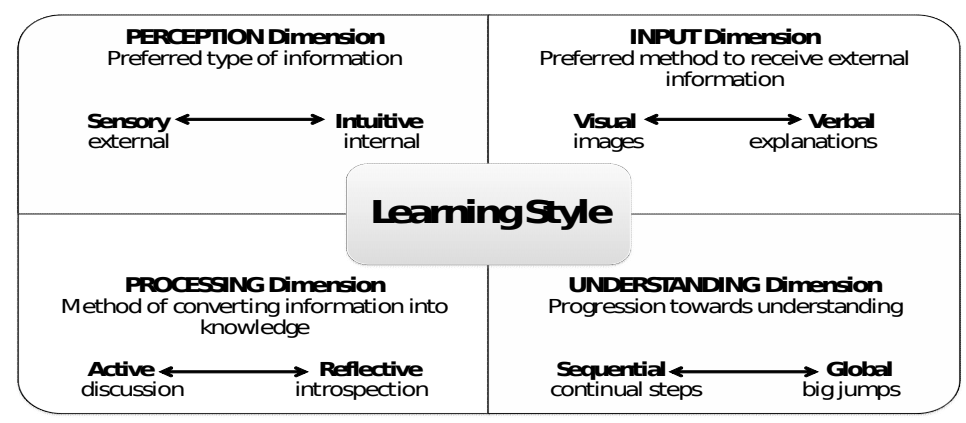

Fig. 1. ILS dimensions.

\subsection{Conversational Agents}

Conversational agents (CAs) are computer programs that allow people to interact with computer systems intuitively using natural language dialogues [13]. CA interfaces have been used effectively to add a 'friendly face' to many applications, such as webbased guidance [14], database interfaces [15] and tutoring [3]. However, CAs are difficult and time-consuming to develop, requiring expertise in the scripting of conversations, and consequently are rarely found in ITS. Intelligent systems such as Oscar CITS which aim to mimic a human tutor need CA interfaces to support the construction of knowledge through discussion [8].

CAs conduct a conversation by accepting natural language user input and producing an appropriate response. Responses usually consist of predefined 'canned text' that can be changed to reflect the conversation context using variables. For example, variables can be used to include a name, e.g. 'How are you feeling today, Bob'. Text-based CAs usually adopt a pattern matching [16] or semantic based [17], [18] approach. Semantic-based CAs seek to understand the meaning of the input by studying the constructs and meanings of natural language [18] or by comparing the semantic similarity of phrases [17]. Pattern-matching CAs rely on a knowledge base containing a set of pattern-based stimulus-response pairs [16] rather than attempting to understand the input.

In the case of Oscar CITS, the pattern matching method was adopted as it can cope with grammatically incomplete or incorrect phrases, as are commonly found in textbased chat by students. The pattern matching approach requires the development of conversation scripts, a similar idea to call centre scripts, which match key input words 
and phrases to suitable responses. Scripts usually contain numerous patterns, leading to many hundreds of stimulus-response pairs in the CA's knowledge base, which demonstrates the complexity and time required to script a CA. Scripts are initially developed by anticipating user utterances and writing stimulus-response pairs to match them. CA scripts require considerable maintenance, needing continuous improvement by reviewing incorrect $\mathrm{CA}$ responses from conversation histories and modifying or adding stimulus-response pairs to address the problem. This requires considerable language expertise, and is labour intensive and time consuming. During a conversation user utterances are matched to stimulus-response pairs in the knowledge base, with the best fitting stimulus-response pair (selected by an algorithm) firing to produce a natural language response.

\subsection{Oscar Conversational Intelligent Tutoring System}

Oscar is an online CITS [19-22], [25] that models a human tutor by dynamically predicting and adapting to each individual student's learning style during a tutoring conversation. Oscar CITS attempts to mimic a human tutor by leading a two-way discussion and using cues from the student dialogue and behaviour to predict and adapt to their learning style. Oscar's intelligent approach includes presenting learning material in the sequence and style most suited to the individual's knowledge and learning style (curriculum sequencing), analysing and giving feedback on incomplete and erroneous solutions (intelligent solution analysis) and giving intelligent hints and discussing questions (problem solving support). Like human tutors, Oscar CITS promotes a deeper understanding of the topic by using a constructivist style of tutoring, giving intelligent hints and discussing questions with learners rather than presenting the answer straight away. Oscar's natural language interface and classroom tutorial style are intuitive to learners, enabling them to draw on experience of face-toface tutoring to feel more comfortable and confident in using the CITS. Oscar CITS is a personal tutor that can answer questions, provide hints and assistance using natural dialogue, and which favours learning material to suit each individual's learning style. The Oscar CITS offers 24-hour personalised learning support at a fixed cost.

In summary, the main features of the innovative Oscar CITS are:

Oscar mimics a human tutor by adopting a tutor-led, conversational approach.

Oscar's natural language interface is intuitive to use and enables learners to actively discuss problems and solutions.

Like human tutors, Oscar supports constructivist learning by incorporating problem solving support and intelligent solution analysis techniques.

Oscar implicitly predicts an individual's learning style by capturing and modelling learner behaviour during a tutoring conversation.

Oscar aims to improve the learning experience by intelligently adapting its tutoring style to match individual's learning styles. 
Oscar CITS is generic, allowing the free choice of learning styles model and subject domain.

Oscar CITS' Automatic Prediction of Learning Styles. The Oscar CITS was initially implemented to deliver a Sequential Query Language (SQL) revision tutorial suitable for undergraduate computing students. The ILS learning styles model was adopted as it describes the learning styles of higher education engineering students. Due to the complexity of the design, development and experimental analysis involved in creating Oscar CITS, the functions of learning style prediction and adaptation were developed separately in two stages. The first implementation (for clarity known hereafter as the Oscar Predictive CITS) incorporated the prediction of learning styles during a tutorial conversation [19]. This paper will describe the implementation of Oscar CITS to incorporate the dynamic adaptation of the conversational tutoring to student learning styles.

The implementation of Oscar Predictive CITS and several initial studies conducted are described in [19-21]. A formal methodology for developing an Oscar Predictive CITS to automatically predict learning styles is detailed in [19] and summarised here. The methodology involves three key phases:

In phase 1, the learning styles predictor agent is created by analysing a learning styles model, mapping typical behaviours to a conversational tutorial style and then representing this knowledge as logic rules that are used to predict learning styles.

In phase 2, the tutorial conversation is designed by capturing a tutorial scenario from human tutors, determining the conversational structure and style by applying templates and then creating CA scripts that are used to conduct the tutorial conversation.

In phase 3, the Oscar Predictive CITS architecture is constructed, and the components (described in section 3.1) are implemented.

For the initial studies investigating the automatic prediction of learning styles, Oscar Predictive CITS delivered an online tutorial in the domain of the database Structured Query Language (SQL). Oscar Predictive CITS draws on knowledge bases of learning styles (the ILS model), tutor material and conversation scripts to deliver a real time conversational tutorial to a student. To support the tutoring conversation, Oscar Predictive CITS may display diagrams, images and interactive movies to aid the student's learning. A number of aspects of the student's behaviour and understanding, such as reading time and the student's approach to answering compound questions, are used to dynamically predict learning style. A report of the detailed process used by Oscar Predictive CITS to model learning styles is given in [19] and [23]. During tutoring the Oscar Predictive CITS records and logs information about each student's behaviour, the tutoring conversation and the student's knowledge of the topic being discussed.

Several experiments were conducted to investigate the success of the Oscar Predictive CITS conversational tutoring and dynamic prediction of learning styles. 
The experiments involved real undergraduate and postgraduate students in a real educational setting. The results show that Oscar Predictive CITS successfully predicted all eight learning styles in the ILS model with accuracies ranging from 61 to $100 \%$ [19-21].

The first implementation of Oscar Predictive CITS successfully incorporated human-like intelligence into a conversational tutorial which improved student test results and dynamically predicted their learning styles [19]. The remainder of this paper will outline the extension of Oscar Predictive CITS to include the ability to adapt a tutorial to an individual student's learning styles.

\section{Oscar: An Adaptive Conversational Intelligent Tutoring System}

Studies in computer-based education systems have shown that adapting the teaching style to student learning styles can enhance learning [24]. Conversational ITS (CITS) are less common than menu-based ITS, and there are no CITS that adapt their teaching to suit an individual's preferred learning styles. Previous work summarised in section 2.4 described Oscar Predictive CITS which leads a real-time tutorial conversation whilst implicitly predicting an individual's preferred learning styles. The contribution of this paper builds on the strengths of Oscar Predictive CITS by incorporating automatic adaptation to each student's learning styles. By adapting the tutoring style to suit individual learners, Oscar Adaptive CITS aims to provide the most appropriate learning material for their learning style, leading to a more effective learning experience and a deeper understanding of the topic.

The following sections describe how the achievements of the Oscar Predictive CITS are used as a basis for designing a CITS that dynamically adapts its teaching style to match student learning styles. The Oscar Adaptive CITS construction is based around delivering preferred teaching styles for individual learners during tutoring. An original generic adaptation algorithm is proposed which takes into account both the strength of each learner's preference and the availability of adaptations to select the best adaptation for each tutorial question.

\subsection{Oscar CITS Architecture}

Typical adaptive educational systems contain student, domain, pedagogical and presentation components [25]. A CITS additionally requires a conversational agent component that can manage a natural language dialogue. The Oscar CITS is independent of a particular learning styles model and of the subject domain being taught. A modular architecture was adopted for Oscar CITS (see Fig. 2) which keeps the system knowledge separate from the functionality, thus allowing the learning styles model and tutoring domain to be changeable. Alternative tutorial knowledge bases and CA scripts developed can be simply 'plugged in' to adapt the tutoring to new subjects. Similarly, different learning styles models may be applied by replacing the Learning Styles Predictor and Learning Styles Adapter agents. The Oscar CITS 
architecture allows individual components to be reused and replaced as necessary, thus minimising tutorial development time.

In the Oscar CITS architecture illustrated in Fig. 2:

the Controller component is the central manager which is responsible for communicating with all components and managing the learner interaction. All communication and information passed between components passes through the controller.

the Graphical User Interface is responsible for display, managing events (such as clicking of buttons etc.) and sending communication to and from the user. As shown in Fig. 3, the display consists of a webpage that provides instructions, displays questionnaires, tests, images, documents, interactive movies and a chat area that is used to communicate with the user. As Oscar CITS directs the tutoring conversation, no navigation buttons are included as there is no menu system.

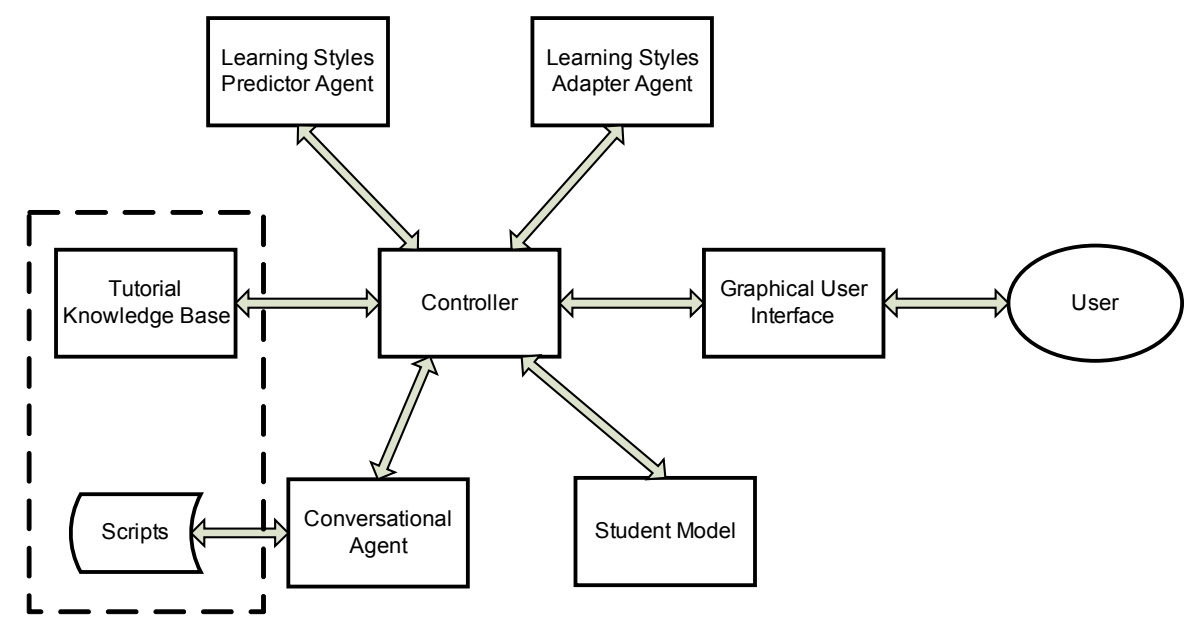

Fig. 2. Oscar CITS architecture.

the Student Model component is a database which maintains all information about individual students, such as their identifier and password, level of knowledge, topics visited, test scores and learning styles. The student model component receives and sends information from and to the controller about the student. The details of the implemented student model structure are given in [23].

the Conversational Agent component is responsible for accepting natural language text and information about topic and learning style from the GUI, tutorial knowledge base and learning styles adapter agents via the controller, and generating a natural language response. The CA accesses a database of conversation scripts (related to but not linked to the tutorial knowledge base) in order to match the input to rules that generate a response. The CA records the dialogue in log files that can be accessed by the controller. 
the Tutorial Knowledge Base component is a database which is responsible for managing course information, such as topics and their breakdowns, related tests and teaching material. All tutor information is related to a particular tutorial module and is categorised according to teaching style (related to learning style). The tutorial knowledge base receives information and instructions from the GUI, learning styles adapter and CA components via the controller, and sends information to the GUI and $\mathrm{CA}$ via the controller. The details of the implemented tutorial knowledge base structure are given in [23].

the Learning Styles Predictor Agent is responsible for analysing information about a student's dialogue and behaviour received from the CA, GUI and student model and predicting the student's learning style. This involves accessing information about learning styles and related teaching styles, held in a learning styles database. The methodology for creating this component was summarised in section 2.5.

the Learning Styles Adapter Agent receives information from the CA, GUI, tutorial knowledge base and student model to select the best adaptation for a student's learning style. Information about learning styles and related teaching styles is accessed from the learning styles database. Given learning style values from the student model and tutorial question scores from the tutorial knowledge base, this agent applies an algorithm to determine the most appropriate adaptation for a student for each individual tutorial question. The methodology for creating this component will be described in section 4 .

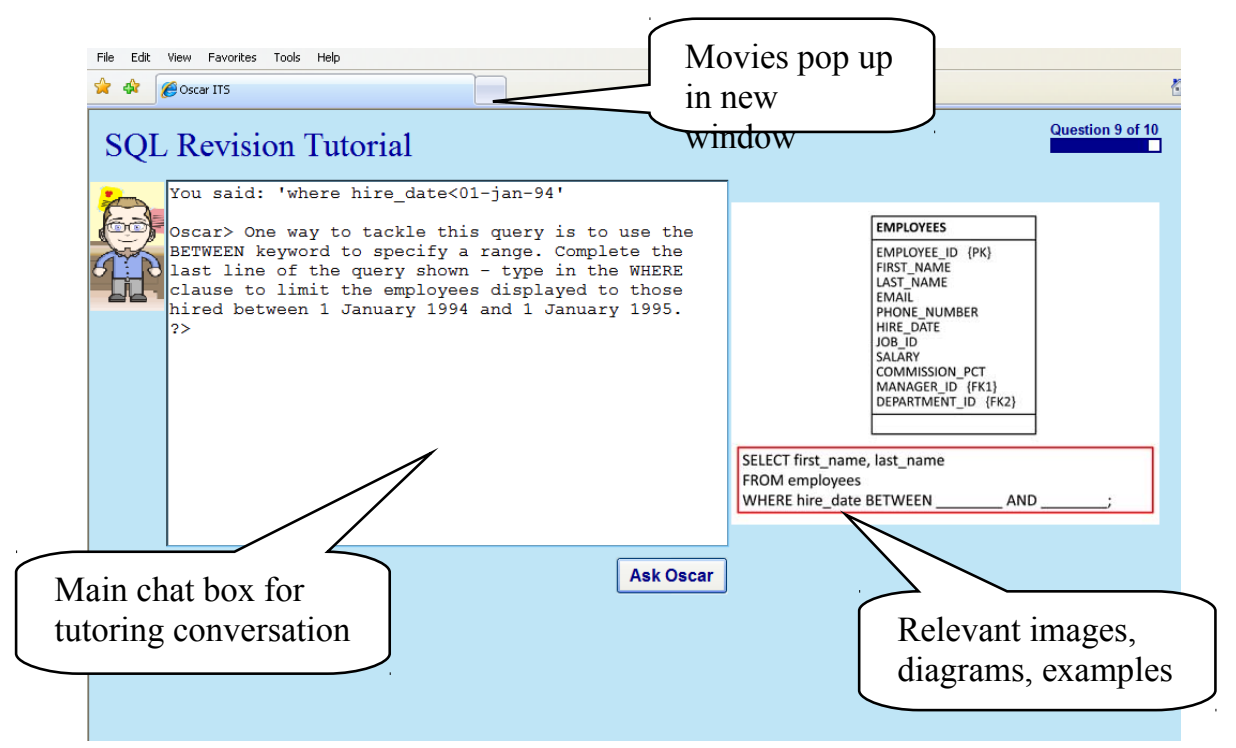

Fig. 3. Oscar CITS.

The implementation of the adaptive Oscar CITS will now be described. 


\section{Methods for Including Adaptivity}

The Oscar Predictive CITS was extended to incorporate the dynamic adaptation of the conversational tutoring style to suit an individual's learning style. Oscar CITS' modular architecture enabled the components developed for previous studies (described in section 3.2) to be reused. A formal three phase methodology for creating an Oscar Adaptive CITS was developed [23]. The methodology is similar but not the same as the methodology for creating the Oscar Predictive CITS that was described in section 2.5. The methodology for creating an Oscar Adaptive CITS is described in [23] and [26] and will now be summarised.

In phase 1 the Learning Styles Adapter Agent is created. First, the Index of Learning Styles model [5] was analysed and a table of learner behaviour for each learning style drawn up. The characteristics were evaluated to establish whether they could be incorporated into a CITS. The subset of learner behaviour considered to be most important for an adaptive CITS was then mapped to the appropriate teaching styles described in the ILS model. The breakdown of behaviour and teaching styles was examined further to develop several domain-independent categories of tutor material required for developing an adaptive CITS, as shown in Table 1. Each tutor material category was mapped to the appropriate learning style, for example, Category 4: Practical Examples maps to the Sensor, Active and Sequential learning styles. The standard categories were designed from the point of view of the tutor and intend to make the development of tutoring material for an adaptive CITS as simple and consistent as possible. The standard organisation of tutor material also facilitates reuse and modular development, as additional materials can be expanded and added without the need for a total redesign of the tutoring session.

Table 1. Teaching material categories mapped to ILS learning styles.

\begin{tabular}{ll}
\hline Teaching Material Category & ILS Learning Styles \\
\hline 1. Introductions and overviews & Sensor, Sequential, Global \\
2. Textual explanation - theories and principles & Intuitor, Verbal, Reflective, Sequential \\
3. Explanation - bullet points and hyperlinks & Intuitor, Global \\
4. Practical examples & Sensor, Active, Sequential \\
5. Practical exercises & Active, Sequential \\
6. Graphics - movies, pictures, diagrams & Intuitor, Visual \\
7. Verbal - movies \& sound clips & Verbal \\
8. Visual demonstrations (walkthroughs etc) & Visual \\
9. Discussion scripts - explanation, FAQs and help & Sensor, Verbal, Active \\
for each subtopic & \\
\hline
\end{tabular}

The final stage in phase 1 was to consider how the Oscar Adaptive CITS would adapt its tutoring according to a student's learning style. The ILS model indicates that students who have no strong learning style preference in a dimension (i.e. they are placed at the centre of the ILS scale with a score of 1 or 3) should be given learning 
material including a mixture of styles. An additional Neutral learning style category was introduced to group those students and a Neutral adaptation style included.

There are a number of possible ways to adapt to learning styles, the simplest of which would be to adapt to the student's strongest learning style. However, a tutorial is made up of a number of tutorial questions, and this approach would require incorporating every category of tutor material into every tutorial question. This may not be possible in real life, as it is important to construct a coherent tutorial and learning experience. Consequently the adaptation strategy needed to consider not only the strength of the student's learning style but also the strength of adaptation available for each individual tutorial question. This strategy ensures that students are presented with the most appropriate tutoring material to suit all aspects of their learning style and the available adaptations for each question in the tutorial. This method was adopted and a complex, domain-independent adaptation algorithm was developed that combined the strengths of the student's learning style with the tutorial adaptations to select the best fitting adaptation for each question in the student's learning path. The novel Oscar CITS adaptation algorithm is described in detail in [23], but a pseudocode representation is shown in Table 2. In Table 2, it is assumed that:

each tutorial question is assigned a score $(\boldsymbol{q})$ for each ILS learning style (8 scores) depending on the strength of adaptation available;

each student is assigned a learning style class $(\boldsymbol{c})$ and score $(\boldsymbol{s})$ for each ILS dimension based on the results of the ILS questionnaire. As defined by the ILS, students with a score of 1 or 3 are assigned a Neutral learning style class for that dimension.

Table 2. Oscar CITS adaptation algorithm.

Assuming that:

1. Each question is assigned a score $\boldsymbol{q}$ for adaptation to each learning style (LS) (8 scores: 2 scores per ILS dimension), based on the number of opportunities in the question to adapt to that learning style.

2. Each student is assigned a learning style class $\boldsymbol{c}$ and score $\boldsymbol{S}$ for each dimension (4 classes, 4 scores) based on the results of the ILS questionnaire. Students with scores of 1 or 3 are assigned the Neutral learning style class. 


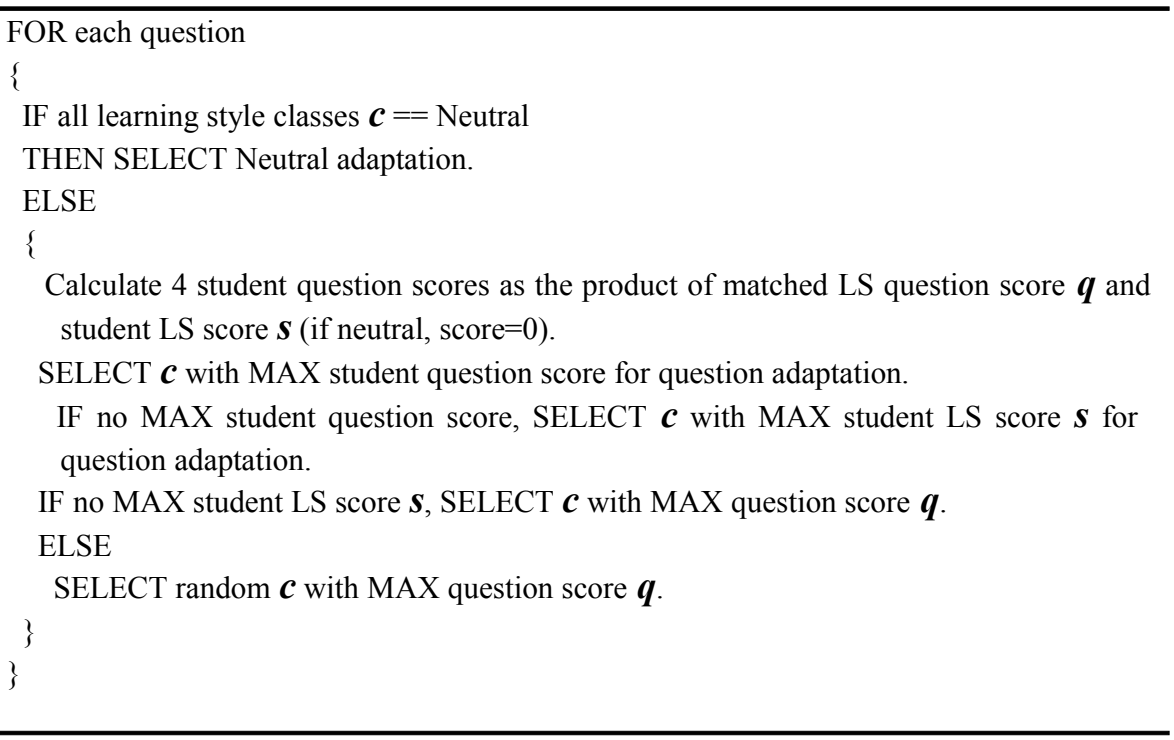

Following the Oscar CITS adaptation algorithm in Table 2, if the student is classed as Neutral for all learning style dimensions they follow the Neutral adaptation learning path for every tutorial question. Otherwise, a student question score is calculated for each learning style dimension by multiplying the question score $(q)$ for the student learning style class (c) with the student learning style score (s). The learning style class $\mathrm{c}$ with the highest student question score is selected for adaptation. Additional rules are included for conflict resolution, i.e. selecting an adaptation when there is more than one maximum student question score, as follows:

Adapt to the student's strongest learning style, i.e. the class $c$ with the highest student learning style score $\boldsymbol{s}$.

If the student has more than one learning style with the maximum score $\boldsymbol{s}$, adapt to the strongest adaptation available for the student's preferred learning styles, i.e. the class $\boldsymbol{c}$ with the highest question score $\boldsymbol{q}$.

Finally, if there is more than one maximum question score $\boldsymbol{q}$, randomly select one of the maximum question scores $\boldsymbol{q}$ and adapt to its learning class $\boldsymbol{c}$.

The implementation of the Oscar CITS adaptation algorithm completes the first phase of developing the Oscar Adaptive CITS, the creation of the Learning Styles Adapter Agent.

In phase 2, the adaptive tutorial conversation is designed. Rather than capturing a new tutorial scenario, it was possible to reuse much of the SQL revision tutorial already developed for the previous implementation of the Oscar Predictive CITS. The adaptive SQL learning material thus extended the tutorial delivered in previous experiments [19-21]. This was achieved by adding different resources covering the standard categories of tutoring material (see Table 1). This involved creating several 
versions of the learning material, each suited to a different learning style. Next, each tutorial question was assigned a score for every learning style which represented the number (or strength) of opportunities for adaptation to that learning style. Where no adaptation existed for a learning style, the question score was zero.

The final step in phase 2 involved creating the conversational agent scripts to conduct the tutoring conversation. The Convagent Ltd. InfoChat CA [27] was adopted as it is a pattern-matching CA that allows information to be captured using variables, and that allows scripts to be organised into different contexts [28]. This ability to organise scripts into contexts is important as to incorporate the adaptation to learning styles, multiple copies of conversation scripts are required which must be organised by learning style adaptation. The CA scripts developed for the SQL revision tutorial were reused and amended to incorporate distinct teaching styles. For example for the Active learning style, CA scripts were amended to replace any detailed explanations of theory with hints based on practical examples and exercises. Overall there were 95 CA contexts (i.e. scripts), containing around 1000 pattern-based stimulus-response pairs written using the InfoChat PatternScript language [29], which demonstrates the complexity of the task of adaptive conversational tutoring. More detail on scripting the CA and an example rule from one of the CA scripts is given in [19] and [23].

The final phase of development (phase 3) involved constructing the Oscar Adaptive CITS architecture in Fig. 2 and described in section 3.1. This involved reusing and amending the components from the Oscar Predictive CITS, as follows:

The GUI was reused without change.

The controller was amended to include additional functionality relating to dynamically selecting adaptations.

The student model component was extended to record adaptations applied for each question.

The conversational agent component was reused without change, although the CA scripts were different, as described in phase 2 .

The tutorial knowledge base was extended to record the adaptation details for each tutorial question, including for each learning style a score and a CA script name.

The learning styles adapter agent was developed as described in phase 1.

The initial study investigating the success of the Oscar Adaptive CITS adaptation to learning styles will now be described.

\section{Experimental Methodology}

An empirical study was conducted using real undergraduate students in a real teaching/learning environment. The study evaluated the success of Oscar Adaptive CITS in adapting its tutoring to individuals' learning styles. The hypothesis was that students who are presented with learning material matched to their learning styles 
perform better than students presented with learning material which is unsuited to their learning styles. Additionally, a pre-test/post-test approach [24] was adopted to measure whether participants have increased their knowledge at the end of the tutorial.

The Oscar Adaptive CITS was implemented to deliver an SQL revision tutorial as described in section 4. Oscar Adaptive CITS is at present installed on a web server and is available via the Internet to Manchester Metropolitan University (MMU) students. The Oscar Adaptive CITS conducts its conversational tutorials in real time and is currently being used in a real teaching/learning environment to support a number of undergraduate and postgraduate computing modules within MMU.

70 final year undergraduate science and engineering students who had previously studied SQL were asked to refresh their SQL knowledge by completing the Oscar Adaptive CITS SQL revision tutorial.

Fig. 4 illustrates the stages involved in the participant interaction with Oscar Adaptive CITS during the study. This involved each participant registering with the Oscar Adaptive CITS anonymously and completing the formal ILS questionnaire before beginning the tutorial. Next, participants completed a pre-tutorial multiple choice question (MCQ) test to assess their existing knowledge before starting the conversational tutorial. The tutorial was led by the Oscar Adaptive CITS tutor which conversed in natural language with participants and guided them through the ten tutorial questions, showing images, movies and examples as necessary. The conversational SQL revision tutorial took on average approximately 43 minutes, with each participant following an individual learning path depending on their knowledge and learning styles (see section 5.1 for example dialogues). After the tutorial conversation, participants completed the same MCQ test and were then presented with a comparison of their test results to indicate learning and some feedback from Oscar. Finally, participants were asked to complete a user evaluation questionnaire.

During the experiment, after completing the ILS questionnaire, participants were unknowingly assigned to one of three experimental groups, as follows:

Participants whose learning styles were at the centre of all ILS scales (i.e. there was no strong preference) were assigned to the Neutral-Adapt group. These participants followed the neutral adaptation learning path, with tutor material including different aspects of all learning styles (e.g. describing theory as well as examples).

Participants who had at least one preferred learning style were randomly assigned to either the Adapt or Mismatch groups using a 2:1 ratio. The restricted randomisation approach was adopted with a 2:1 ratio to attain a balance of participant learning style characteristics as the sample of participants would be small [30]. The participants in these two groups followed an adaptive learning path assigned by the algorithm, and were given tutor material favouring particular learning styles (e.g. containing explanations of theory rather than practical examples). Participants in the Mismatch group were deliberately presented with learning material unsuited to their learning styles, e.g. if a participant was Sequential, they were presented with material suited to a Global participant. 


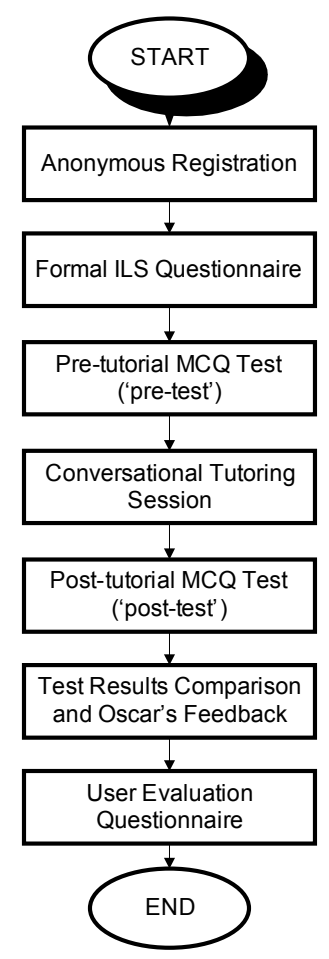

Fig. 4. Stages in the Tutorial Interaction during the Experiment

A match/mismatch approach [31] was chosen rather than an approach where one control group experiences a basic unadapted tutorial. This approach was concluded to be a better test of the adaptation as an experimental group experiencing additional learning material to the control group is likely to show better learning.

For the purpose of the experiment, log files that recorded the dialogue, timings, knowledge and other behavioural factors were analysed for each completed tutorial to assess each participant's performance during the tutoring session. During the SQL Revision tutoring session, ten questions were posed, requiring eighteen answers (as some questions incorporated multiple steps or questions). To test the hypothesis, learning gain was calculated by counting the number of correct answers given to tutorial questions, and an average score was calculated for each experimental group. The experimental group averages were then compared to determine whether there was any difference in learning gain related to the type of adaptation to learning style. In addition, Oscar Adaptive CITS' ability to tutor effectively was evaluated by analysing the user feedback questionnaires and test score improvements. 


\section{Results and Discussion}

Of the 70 participants, 54 fully completed the tutoring session. Table 3 shows the results of the experiment. The group of 8 Neutral-Adapt participants had learning style results that showed no strong preference for a particular learning style (i.e. their styles were balanced in the centre of the scale), and followed a neutral adaptation learning path. The Adapt group contained 32 participants who followed a learning path containing learning material in a style adapted to their individual learning styles. The Mismatch group of 14 participants followed an adaptive learning path of tutor material that was mismatched to their learning styles.

Table 3. Experimental results.

\begin{tabular}{llcccc}
\hline $\begin{array}{c}\text { Experimental } \\
\text { Group }\end{array}$ & $\mathbf{n}$ & \multicolumn{2}{c}{$\begin{array}{c}\text { Mean Correct Answers } \\
\mathbf{~} \mathbf{1 8}\end{array}$} & SD & Median \\
\hline Neutral-Adapt & 8 & 12.875 & $72 \%$ & 2.416 & 13 \\
Adapt & 32 & 13.188 & $73 \%$ & 2.101 & 13 \\
Mismatch & 14 & 11.000 & $61 \%$ & 3.258 & 11 \\
\hline Total & 54 & 12.574 & $70 \%$ & 2.618 & 13 \\
\hline
\end{tabular}

Participants in the Neutral-Adapt and Adapt groups have similar averages of correct answers given during the tutoring, of $72 \%$ and $73 \%$ respectively. However, the Mismatch group has a much lower average of only $61 \%$ correct answers, which is $12 \%$ less than the Adapt group average. The Kruskal-Wallis statistical test [32] was applied with a 95\% significance level as it indicates whether or not distributions are the same, but does not require normality. The Kruskal-Wallis test results gave a Kruskal-Wallis statistic of 7.03 and a p-value of 0.03 , showing that there is a significant difference in the Mismatch group. The results support the hypothesis that participants who are presented with learning material matched to their learning styles perform significantly better than participants presented with learning material that is not matched to their learning styles.

In general, the user evaluation results showed that the Oscar Adaptive CITS was well received, with $95 \%$ of participants finding the tutoring helpful and $89 \%$ agreeing that Oscar helped them to revise. $91 \%$ of the sample said that they would use the Oscar Adaptive CITS resource, with $86 \%$ stating they would use Oscar to support classroom tutoring and $77 \%$ saying they would use Oscar instead of learning from a book. A surprising 50\% of the sample said they would use Oscar in place of attending face-to-face tutorials. There was an overall mean test score improvement after tutoring of $19 \%$, showing that participants did learn from the tutoring.

A comparison with other CITS is not possible, as no other CITS can adapt their tutoring style to match an individual's learning styles. 


\subsection{Examples of Adaptive Learner Dialogues}

Examples of the Oscar Adaptive CITS tutoring style adaptation can be seen in the dialogue snippets logged during the experiment (Tables 4 and 5). Both snippets are taken from the same tutorial question where participants are asked to write an SQL query, and both participants were assigned to the Adapt experimental group (i.e. the adaptation matched their learning style). The participant dialogue in Table 4 is an example of the tutoring style adapted to a Global learner, where the participant writes a query without initial guidance and learns from the feedback given about errors in their solution. Table 5 in contrast shows a participant dialogue for the same question with tutoring adapted for a Sequential learner, where Oscar Adaptive CITS guides the participant through the steps of the query.

Table 4. Dialogue snippet logged during the experiment adapting to a Global learner.

\begin{tabular}{|c|c|}
\hline Utterance & Dialogue \\
\hline 64. Oscar> & $\begin{array}{l}\text { Now let's see if you can remember how to write a query which chooses } \\
\text { values between a range. Look at the employees table. Please type in a query } \\
\text { which displays the first and last names of all employees hired between } 1 \\
\text { January } 1994 \text { and } 1 \text { January } 1995 \text {. }\end{array}$ \\
\hline 65. Student> & $\begin{array}{l}\text { select First_name, last_name from Employees where Hire_date between 1- } \\
\text { JAN-1944 and 1-Jan-1995; }\end{array}$ \\
\hline 66. Oscar> & $\begin{array}{l}\text { I'm afraid your WHERE clause is not quite right. Check your spelling and } \\
\text { type in the last part of our query which displays the first and last names of } \\
\text { all employees hired between } 1 \text { January } 1994 \text { and } 1 \text { January } 1995 \text {. }\end{array}$ \\
\hline 67. Student> & $\begin{array}{l}\text { select First_name, last_name from Employees where Hire_date between 1- } \\
\text { JAN-1994 and 1-Jan-1995; }\end{array}$ \\
\hline 68. Oscar> & $\begin{array}{l}\text { No. Excluding date conversion functions, ORACLE recognises dates in } 3 \\
\text { basic formats: '01-JAN-94', '01-January-94' and '01-January-1994'. The } \\
\text { picture shows two different ways in which the WHERE clause could have } \\
\text { been written to give the right results. }\end{array}$ \\
\hline
\end{tabular}

Table 5. Dialogue snippet logged during the experiment adapting to a Sequential learner.

\begin{tabular}{|c|c|}
\hline Utterance & Dialogue \\
\hline 87. Oscar> & $\begin{array}{l}\text { Now let's see if you can remember how to write a query which chooses } \\
\text { values between a range. Look at the employees table. We need a query } \\
\text { which displays the first and last names of all employees hired between } 1 \\
\text { January } 1994 \text { and } 1 \text { January } 1995 \text {. The first step in writing a query is to state } \\
\text { the information you need. For our query to display the first and last names } \\
\text { of all employees hired between } 1 \text { January } 1994 \text { and } 1 \text { January 1995, which } \\
\text { columns do we need? }\end{array}$ \\
\hline 88. Student $>$ & dont know \\
\hline 89. Oscar> & $\begin{array}{l}\text { Look again at the columns in the EMPLOYEES table. If we want to display } \\
\text { the first and last names of all employees hired between } 1 \text { January } 1994 \text { and } \\
1 \text { January 1995, which columns do we need? }\end{array}$ \\
\hline
\end{tabular}


91. Oscar> That's right, we need FIRST NAME and LAST NAME, so the first part of our query is shown in the picture. The next step in writing a query is to state which table the information is retrieved from. For our query to list the first and last names of all employees hired between 1 January 1994 and 1 January 1995, type in the name of the table our information will be taken from.

92. Student> employees

93. Oscar> That's right, the table is EMPLOYEES. The last part of writing a query is the WHERE clause, which lets us restrict the rows selected to only those satisfying one or more conditions. We want to restrict the employees displayed to only those hired between 1 January 1994 and 1 January 1995. There are a number of ways to write this restriction in ORACLE SQL as dates can be represented in different formats. Type in the WHERE clause which limits the employees in this way.

94. Student> where hire date in('01-01-1994' \& \& '01-01-1995')

95. Oscar> One way to tackle this query is to use the BETWEEN keyword to specify a range. Complete the last line of the query shown - type in the WHERE clause to limit the employees displayed to those hired between 1 January 1994 and 1 January 1995.

96. Student> '01-01-1994' and '01-01-1995'

97. Oscar> OK, excluding date conversion functions, ORACLE recognises dates in 3 basic formats: '01-JAN-94', '01-January-94' and '01-January-1994'. The picture shows two different ways in which the WHERE clause could have been written to give the right results.

\section{Conclusions and Further Work}

In this paper a new version of Oscar CITS has been described, which is a novel conversational intelligent tutoring system that implicitly adapts to an individual's learning style. The research built on previous work in which Oscar Predictive CITS can implicitly predict a person's learning styles based on their behaviour during a tutoring conversation [19-22]. The Oscar Adaptive CITS described here imitates a human tutor by conversing with students and delivering learning material adapted to their learning styles, individualised problem solving support and intelligent solution analysis. A CITS that personalises tutoring by dynamically predicting and adapting to learning styles could improve the effectiveness of a student's learning experience and help to boost confidence. Effective, personalised online tutoring can flexibly support class-based courses and also widen access through distance learning.

This paper describes how the adaptation to learning styles was introduced into Oscar CITS, and presents the Oscar CITS adaptation algorithm. Unlike other adaptive tutoring systems, the Oscar CITS algorithm considers both the strength of an individual's learning style preferences and the availability of adaptive tutor material.

A study is presented, which investigates the success of the Oscar CITS adaptation algorithm. The results of the study showed that participants whose learning path adapted to their learning styles performed significantly better, achieving on average $12 \%$ more correct answers, than those participants presented with learning material not matched to their learning styles. Additionally, participants valued Oscar's conversational tutoring, which seemed to help learning with test scores improved by 
$19 \%$ on average. It can therefore be concluded that using Oscar CITS has helped provide students with a positive learning experience.

In future, it is planned to incorporate the tutor material categories into a development toolkit to speed up the scripting of an adaptive CITS.

Acknowledgement. The research presented in this paper was funded by EPSRC. The authors thank Convagent Ltd for the use of the InfoChat conversational agent and PatternScript scripting language.

\section{References}

Brusilovsky, P., Peylo, C.: Adaptive and Intelligent Web-based Educational Systems. Int. J. Artificial Intelligence in Education 13, pp. 156--169 (2003)

D’Mello, S., Lehman, B., Sullins, J., Daigle, R., Combs, R., Vogt, K., Perkins, L., Graesser, A.: A Time for Emoting: When Affect-Sensitivity Is and Isn't Effective at Promoting Deep Learning. In ITS 2010, LNCS, vol. 6094, pp. 245--254, Springer, Heidelberg (2010)

Graesser, A., Chipman, P., Haynes, B.C., Olney, A.: AutoTutor: An Intelligent Tutoring System With Mixed-Initiative Dialogue. IEEE Trans. Education 48 (4), pp. 612--618 (2005)

Popescu, E.: Adaptation provisioning with respect to learning styles in a Web-based educational system: an experimental study. J. Computer Assisted Learning 26, pp. 243--257 (2010)

Felder, R., Silverman, L.K.: Learning and Teaching Styles in Engineering Education. J. Engineering Education 78 (7), 674--681 (1988)

Cha, H. J., Kim, Y. S., Park, S. H., Yoon, T. B., Jung, Y. M., Lee, J. H.: Learning styles diagnosis based on user interface behaviours for the customization of learning interfaces in an intelligent tutoring system. In: ITS 2006, LNCS, vol. 4053, pp. 513--524, Springer, Heidelberg (2006)

Klasnja-Milicevic, A., Vesin, B., Ivanovic, M., Budimac, Z.: E-Learning personalization based on hybrid recommendation strategy and learning style identification. Computers \& Education 56, pp. 885--899 (2011)

Chi, M.T.H., Siler, S., Jeong, H., Yamauchi, T., Hausmann, R.G.: Learning from human tutoring. Cognitive Science 25, pp. 471--533 (2001)

Woo Woo, C., Evens, M.W., Freedman, R., Glass, M., Seop Shim, L., Zhang, Y., Zhou, Y., Michael, J.: An intelligent tutoring system that generates a natural language dialogue using dynamic multi-level planning. Artificial Intelligence in Medicine 38, pp. 25--46 (2006)

Mitrovic, A.: An Intelligent SQL Tutor on the Web. Int. J. Artificial Intelligence in Education 13, pp. 171--195 (2003)

Ammar, M. B., Neji, M., Alimi, A. M., Gouarderes, G.: The Affective Tutoring System. Expert Systems with Applications 37, 3013--3023 (2010)

Leontidis, M., Halatsis, C.: Integrating Learning Styles and Personality Traits into an Affective Model to Support Learner's Learning. In: Spaniol, M. et al. (Eds.) ICWL 2009. LNCS, vol. 5686, pp. 225--234. Springer, Heidelberg (2009)

O'Shea, J., Bandar, Z., Crockett, K.: Systems Engineering and Conversational Agents. In A. Tolk \& L.C. Jain (eds.), Intelligence-based Systems Engineering, Intelligent Systems Reference Library, vol. 10, Springer, Heidelberg (2011)

Latham, A., Crockett, K., Bandar, Z.: A Conversational Expert System Supporting Bullying and Harassment Policies. In: Proc. ICAART 2010, pp. 163--168 (2010). 
Owda, M., Bandar, Z., Crockett, K.: Information Extraction for SQL Query Generation in the Conversation-Based Interfaces to Relational Databases (C-BIRD). In: KES AMSTA 2011, LNAI, vol. 6682, pp. 44--53, Springer, Heidelberg (2011)

Michie, D.: Return of the Imitation Game. Electronic Transactions on Artificial Intelligence 6, pp. 203--221 (2001)

Li, Y., Bandar, Z., McLean, D., O'Shea, J.: A Method for Measuring Sentence Similarity and its Application to Conversational Agents. In Proc. FLAIRS 2004, pp. 820--825 (2004)

Khoury, R., Karray, F., Kamel, M.S.: Keyword extraction rules based on a part-of-speech hierarchy. Int. J. Advanced Media and Communication 2 (2), pp. 138--153 (2008)

Latham, A.M., Crockett, K.A., McLean, D.A., Edmonds, B.: A conversational intelligent tutoring system to automatically predict learning styles. Computers \& Education 59, pp. 95-109 (2012)

Latham, A.M., Crockett, K.A., McLean, D.A., Edmonds, B.: Predicting Learning Styles in a Conversational Intelligent Tutoring System. In X. Luo et al (eds.), ICWL 2010, LNCS, vol. 6483, pp. 131--140, Springer (2010)

Latham, A.M., Crockett, K.A., McLean, D.A., Edmonds, B., O'Shea, K.: Oscar: An Intelligent Conversational Agent Tutor to Estimate Learning Styles. In Proc. IEEE World Congress On Computational Intelligence 2010, pp. 2533--2540 (2010)

Latham, A.: Personalising Learning with Dynamic Prediction and Adaptation to Learning Styles in a Conversational Intelligent Tutoring System. PhD Thesis, Manchester Metropolitan University (2011)

Kelly, D., Tangney, B.: Adapting to intelligence profile in an adaptive educational system. Interacting with Computers 18, pp. 385--409 (2006)

Wenger, E.: Artificial Intelligence and Tutoring Systems. Morgan Kaufmann, Los Altos, CA (1987)

Latham, A.M., Crockett, K.A., McLean, D.A., Edmonds, B.: Oscar: An Intelligent Adaptive Conversational Agent Tutoring System. In: KES AMSTA 2011, LNAI, vol. 6682, pp. 563-572, Springer (2011)

Convagent Ltd.: Convagent. [Accessed on 17 May 2011] http://www.convagent.com/ (2005)

Sammut, C.: Managing Context in a Conversational Agent. Linkoping Electronic Articles in Computer \& Information Science 3 (7). Linkoping University Electronic Press, Sweden (2001)

Michie, D., Sammut, C.: Infochat Scripter's Manual. ConvAgent Ltd., Manchester, UK (2001)

Schultz, K.F., Grimes, D.A.: Generation of allocation sequences in randomised trials: chance, not choice. The Lancet 359, pp. 515--519 (2002)

Tsianos, N., Lekkas, Z., Germanakos, P., Mourlas, C., Samaras, G.: User-centred Profiling on the basis of Cognitive and Emotional Characteristics: An Empirical Study. LNCS, vol. 5149, pp. 214--223 (2008)

Kruskal, W., Wallis, W.A.: Use of ranks in one-criterion variance analysis. J. American Statistical Association 47 (260), pp. 583--621 (1952) 